\title{
Herbicidas en la milpa: Estrategias de aplicación y su impacto sobre el consumo de arvenses
}

\section{Herbicide milpa: Application strategy and its impact on edible weeds consumption}

\author{
Ricardo Daniel Mascorro-de Loera ${ }^{1 *}$, Bruce G. Ferguson ${ }^{10}$, Hugo Rafael Perales-Rivera1 이, Fabien \\ Charbonnier ${ }^{1}$ \\ ${ }^{1}$ El Colegio de la Frontera Sur. Carretera Panamericana y Periférico Sur s/n, Barrio Ma. Auxiliadora, San Cristóbal de las Casas, \\ Chiapas, México. \\ *Autor de correspondencia: daniel.mascorro@gmail.com
}

Artículo científico recibido: 13 de diciembre de 2018 aceptado: 30 de abril de 2019

RESUMEN. Las arvenses son plantas que crecen de forma espontánea en áreas agrícolas, y algunas forman parte de la alimentación campesina. Los estudios indican que el uso prolongado de herbicidas disminuye la disponibilidad de arvenses y reduce su consumo. El objetivo fue conocer las estrategias de aplicación de los herbicidas y cómo afectan el consumo de arvenses en el municipio de Amatenango del Valle, Chiapas. Se realizaron entrevistas abiertas y se visitaron parcelas para conocer el uso de herbicidas y la disponibilidad de arvenses para consumo. Se aplicaron cuestionarios a 40 hogares para documentar el conocimiento de las arvenses comestibles, su consumo actual y el de hace 10 años. Todos los agricultores usan herbicidas, en promedio tienen $24( \pm 11)$ años aplicándolo. Los entrevistados alternan la aplicación de herbicida con azadón y machete, el herbicida lo aplican de forma selectiva y mantienen zonas de refugio. Estas prácticas contribuyen a la persistencia de las arvenses; los solares, la milpa de riego y la compra son sitios alternos de obtención de arvenses. Los entrevistados consumen arvenses pero perciben que el consumo de Amaranthus hybridus, Galinsoga quadriradiata, Brassica rapa, Crotalaria longirostrata y Physalis philadelphica ha disminuido en los últimos diez años $(p<0.05)$. Esta percepción no está asociada con el número de fumigaciones ni los años de aplicación $\left(\mathrm{Chi}^{2}, \mathrm{p}>0.05\right)$. A pesar de los cambios en su dieta por influencia de alimentos industrializados y cambios tecnológicos en el manejo de la milpa, las arvenses continúan siendo un elemento arraigado en la gastronomía de Amatenango del Valle.

Palabras clave: Agricultura tradicional, alimentación, cambio tecnológico, etnobotánica, gastronomía local.

ABSTRACT. Many edible weeds growing spontaneously in agricultural areas form part of peasant diets. Some studies indicate that long-term herbicide use diminishes edible weed availability, and presumably, consumption. The research presented here sought to understand the application strategy between herbicide use and the impact on edible weed consumption in the municipality of Amatenango del Valle, Chiapas. Open interviews and plot visits were performed to explore herbicide and edible weed use. Forty farm households were surveyed regarding knowledge and consumption of edible weeds. All of those surveyed apply herbicide and have done so for an average $24( \pm 11)$ years. However they alternate between herbicide application and manual control with hoes and machetes, apply herbicides selectively, and maintain herbicide-free refuges. These practices seem to permit the persistence of edible weeds. They also obtain weeds from home gardens, irrigated fields, and markets. The interviewees continue to consume a variety of weeds, but perceive that their consumption of Amaranthus hybridus, Galinsoga quadriradiata, Brassica rapa, Crotalaria longirostrata and Physalis philadelphica has diminished over the last 10 years $(p<0.05)$. This decrease is not associated with the number of with herbicide applications or the number of years in which herbicides have been used $\left(\mathrm{Chi}^{2}, \mathrm{p}>0.05\right)$. Despite the incipient industrialization of their diet and technological changes in agricultural production, edible weeds maintain their importance in the gastronomy of Amatenango del Valle.

Key words: Traditional farming, diet, technological change, ethnobotany, local gastronomy. 
Mascorro-de Loera et al.

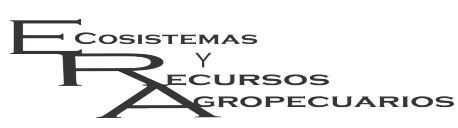

Herbicidas e impacto en la milpa

Ecosist. Recur. Agropec. 6(18):477-486,2019

\section{INTRODUCCIÓN}

Se denomina arvenses o quelites a las plantas que crecen de forma espontánea en áreas agrícolas (Bourges et al. 2015, Mera et al. 2005). Su presencia se asocia a las actividades humanas como resultado de su adaptación a los ambientes creados por el manejo (Bye 1981). Sus usos pueden ser alimenticio, ritual, medicinal, forrajero, para construcción o como abono (Albino-García et al. 2011, SánchezBlanco y Guevara-Féfer 2013) por lo que las arvenses forman parte importante de la dieta de las familias campesinas (Silva et al. 2003), y proporcionar insumos para la cocina (Buenrostro 2009). Además, son de bajo costo y tienen alta disponibilidad (Caballero et al. 2011). Proveen ventajas para la salud (Blanckaert et al. 2007), en parte por su aporte de vitaminas, minerales y otros nutrientes (Caballero et al. 1998). Fungen como reserva de alimentos o alimentos de emergencia (Vázquez-García et al. 2004), y por lo tanto contribuyen a la seguridad alimentaria (Linares y Bye 2015). Cuando hay excedentes las familias las comercializan para obtener recursos económicos adicionales (Carrera-García et al. 2012, González-Amaro et al. 2009).

Estudios en distintos países indican la importancia de las arvenses en la alimentación y gastronomía local (Sinha y Lakra 2007, Turreira-García et al. 2015). En la milpa, policultivo que integra al maíz (Zea mays L.), frijol (Phaseolus sp.) y calabaza (Curcubita sp.) (Linares y Bye 2011, 2015, ZizumboVillarreal y Colunga-García 2010); se reportan alrededor de 127 arvenses comestibles (Linares y Bye 2015). Para el estado de Chiapas, se reportan 39 especies de arvenses comestibles asociadas a la milpa, distribuidas en 17 familias, 29 géneros y una especie no identificada (Mariaca et al. 2007, Ramírez-Salinas y Castro-Ramírez 2011, Solís-Becerra y EstradaLugo 2014). Para Amatenango del Valle y Teopisca, Chiapas, se reportaron 14 especies de arvenses comestibles en el maíz (Ramírez-Salinas y CastroRamírez 2011, Solís-Becerra y Estrada-Lugo 2014)

La milpa se siembra con base en la agricultura tradicional bajo el sistema roza-tumba - quema y se mantiene el cultivo a través del uso del fuego, la acción humana y herramientas simples (Hernández 1985, Lara et al. 2012). Las herramientas comúnmente utilizadas para el control de arvenses, junto con el deshierbe manual, son el machete y azadón. Desde la década de los 1940 con la Revolución Verde se comenzó el uso masivo de herbicidas (Sonnenfeld 1992). Los herbicidas pueden ser selectivos como el 2-4-D para especies de hoja ancha o no selectivos como paraquat o glifosato que eliminan a todas las plantas (Viveros 2005). El uso de herbicidas ha ocasionado cambios drásticos en el manejo de la milpa tradicional (Vigouroux et al. 2011) y las comunidades de arvenses disminuyen su diversidad (Chikoye et al. 2004, Grundy et al. 2011). En el estado de Chiapas los herbicidas se usan ampliamente (Bernardino et al. 2014), siendo el segundo estado, después de Sinaloa, con mayor uso de herbicidas (Albert 2005). Su aplicación puede o no acompañarse con el uso de machete o azadón, por ejemplo en Amatenango del Valle el $86.0 \%$ de los campesinos usan herbicidas (Bernardino et al. 2014). Por lo anterior, el objetivo de la investigación fue identificar los cambios en el consumo de arvenses y su posible disminución a causa del uso de herbicidas.

\section{MATERIALES Y MÉTODOS}

El trabajo se realizó en Amatenango del Valle, Chiapas, municipio indígena con lengua Tseltal. Se ubica a $17^{\circ} 32^{\prime} \mathrm{LN}$ y $92^{\circ} 26^{\prime} \mathrm{LO}$, forma parte de la región de Los Altos de Chiapas (Figura 1). Los sitios de estudio se ubican entre 1850 y $2100 \mathrm{msnm}$. El clima es templado subhúmedo con lluvias en verano (INEGI 2005). Su principal actividad agrícola es la siembra de maíz, con una superficie total de 2369 ha de las cuales 2237 ha son de temporal (SIAP 2017).

Para el trabajo de campo, se solicitó permiso a las autoridades municipales y tradicionales. La unidad de análisis fueron los jefes(as) de familia. Se aplicaron 40 encuestas de modo aleatorio entre abril y julio de 2016 a personas mayores de 18 años. El diseño de la encuesta se fundamentó con lo propuesto por Ladio y Lozada (2004) y, Ramírez-Salinas y Castro-Ramírez (2011) que consiste en mostrar un catálogo fotográfico de los ar- 


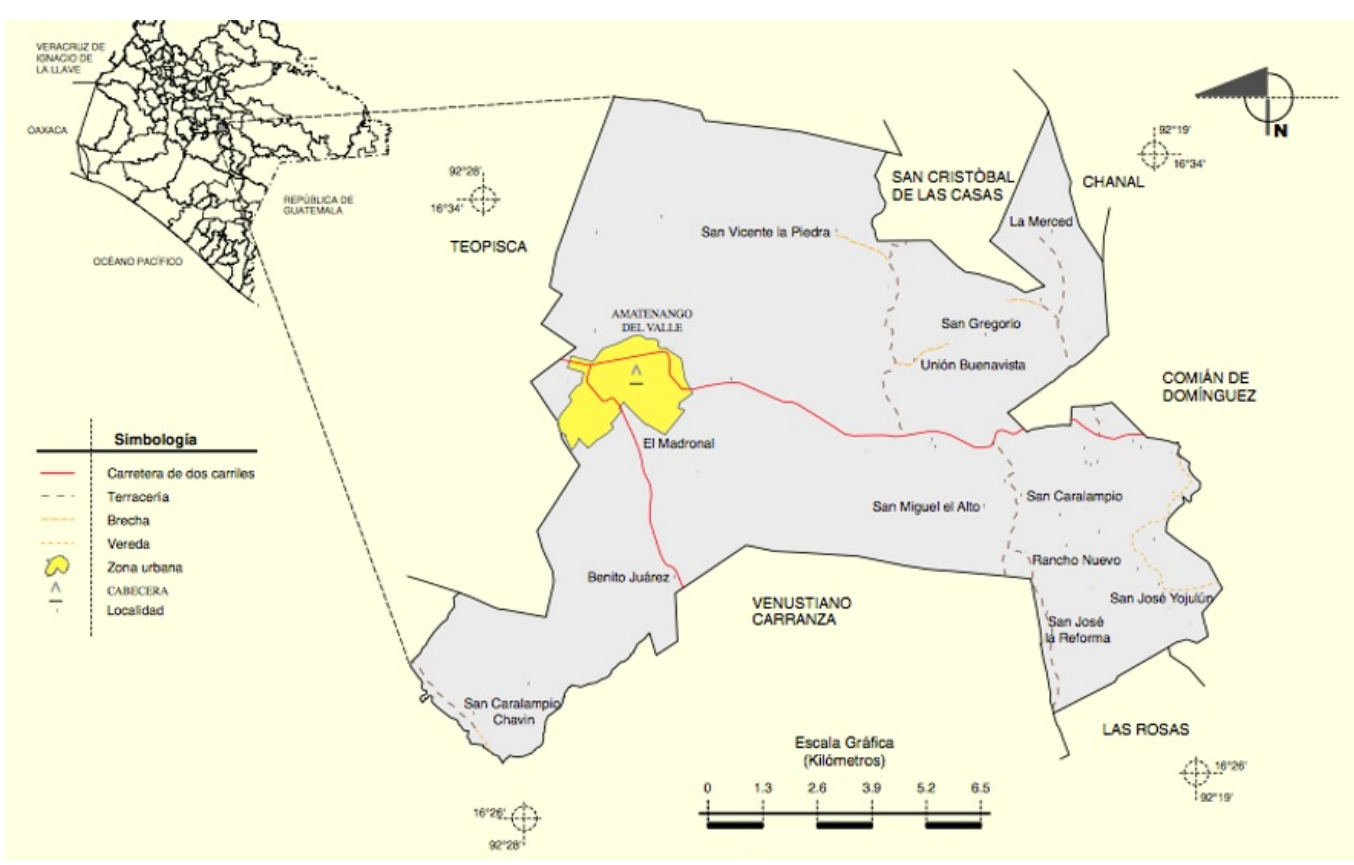

Figura 1. Localización de la zona de estudio.

venses seguido por un cuestionario, que contenía los siguientes rubros; información socioeconómica, manejo de la milpa, etnobotánica y consumo de los arvenses tomando en cuenta la temporalidad, presencia o ausencia en la milpa y frecuencia de consumo actual e histórico. El catálogo fotográfico contempló 13 de las 14 especies reportadas en Teopisca y Amatenango del Valle por RamírezSalinas y Castro-Ramírez (2011) y, Solís-Becerra y Estrada-Lugo (2014) debido a la cercanía de ambas comunidades (Tabla 1). De manera paralela a 10 jefes(as) de familia que lo permitieron se acompañaron a las labores agrícolas con el fin de realizar observaciones directas en la aplicación de herbicida, manejo y consumo de arvenses.

El análisis de los datos se realizó con pruebas estadísticas paramétricas y no paramétricas utilizado el paquete estadístico SPSS versión 20.0.0.0. Para determinar diferencias significativas en la frecuencia de consumo de arvenses actual e histórico se aplicó la prueba de $t$ para muestras relacionadas con transformación a logaritmo natural. Mientras que para determinar la independencia de los datos entre la percepción de consumo de arvenses y el uso de her- bicidas se aplicó la prueba Chi cuadrada.

\section{RESULTADOS}

De los jefes de familia encuestados $(n=40)$, $10 \%$ fueron mujeres y $90 \%$ hombres. La edad mínima fue de 20 años y la máxima de 89 , con edad promedio de 47 años $( \pm 14.7)$. La actividad principal es la agricultura (93.9\%). El 87.5\% sembró maíz el año pasado de los cuales el $68.6 \%$ cuentan con terrenos de riego y temporal, el $25.7 \%$ sólo temporal y el $5.7 \%$ restante sólo riego. Los resultados presentados corresponden solo a los jefes de familia que el 2015 sembraron maíz en terreno de temporal $(n=33)$.

\section{Manejo de la milpa}

No se encontraron siembras de maíz en forma de monocultivo. El $27.3 \%$ siembra maíz asociado con frijol y el $72.7 \%$ restante siembran maíz asociado con frijol (Phaseolus spp.) y Curcubita spp. como calabaza y chilacayote. El control de arvenses previo a la siembra se realiza principalmente de forma mecánica, del total el $87.9 \%$ utiliza el fuego o chaporreo con machete en combinación o de manera ais- 
Mascorro-de Loera et al.

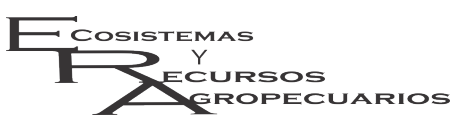

Tabla 1. Consumo y siembra de arvenses en la milpa de temporal (porcentaje, $\mathrm{n}=33$ ).

\begin{tabular}{lccccc}
\hline Especie & $\begin{array}{c}\text { Se requiere } \\
\text { sembrar }\end{array}$ & $\begin{array}{c}\text { Presencia en } \\
\text { milpa de temporal }\end{array}$ & $\begin{array}{c}\text { Comió el } \\
\text { año pasado }\end{array}$ & $\begin{array}{c}\text { Hace 10 años } \\
\text { la comía }\end{array}$ & Variación \\
\hline Amaranthus hybridus $^{1}$ & 0.0 & 93.9 & 93.9 & 97.0 & -3.1 \\
Galinsoga quadriradiata $^{2}$ & 0.0 & 97.0 & 100.0 & 100.0 & 0.0 \\
Sonchus oleraceus $^{1,2}$ & 0.0 & 93.9 & 100.0 & 100.0 & 0.0 \\
Brassica juncea $^{1}$ & 66.7 & 45.5 & 93.9 & 100.0 & -6.1 \\
Brassica rapa $^{1}$ & 63.6 & 45.5 & 100.0 & 100.0 & 0.0 \\
Chenopodium ambosioides $^{1}$ & 6.1 & 21.2 & 100.0 & 100.0 & 0.0 \\
Ipomoea purpurea $^{1,2}$ & 0.0 & 81.8 & 3.0 & 6.1 & -3.1 \\
Crotalaria longirostrata $^{1}$ & 21.2 & 42.4 & 97.0 & 100.0 & -3.0 \\
Oxalis corniculata $^{2}$ & 0.0 & 72.7 & 42.4 & 84.8 & -42.4 \\
Portulaca oleracea $^{1}$ & 3.0 & 78.8 & 60.5 & 63.6 & -3.1 \\
Jaltomata procumbens $^{1,2}$ & 0.0 & 42.4 & 9.1 & 12.1 & -3.0 \\
Solanum nigrescens $^{1}$ & 3.0 & 75.8 & 42.4 & 48.5 & -6.1 \\
Physalis philadelphica $^{1,2}$ & 9.1 & 72.7 & 100.0 & 100.0 & 0.0 \\
\hline${ }^{1}$ Reportada en el municipio de Teopisca. $^{2}$ Reportada en el municipio de Amatenango del Valle. &
\end{tabular}

lada, mientras que el $12.1 \%$ combina el uso de machete y herbicida.

Una vez sembrado el maíz, los herbicidas sustituyen el uso de medios mecánicos por la aplicación de herbicida. El $15.2 \%$ de los encuestados realiza dos deshierbes, el $78.7 \%$ tres y el $6.1 \%$ cuatro. En el primer deshierbe sólo el $6.1 \%$ combina el uso de machete con herbicida, mientras que el $93.9 \%$ aplica sólo herbicida. En el segundo deshierbe sólo el 3.0\% utilizó azadón, el 9.1\% combina azadón y herbicida y, el $87.9 \%$ restante sólo herbicida. De los campesinos que realizan tres deshierbes, el 3.7\% combina el uso de azadón o machete con herbicida y el $96.3 \%$ restante aplica sólo herbicida. En tanto que los campesinos que realizan hasta cuatro deshierbes sólo aplican herbicida.

Los herbicidas más utilizados son el glifosato, paraquat y 2-4-D bajo una gran gama de nombres comerciales. El tiempo promedio que se han usado es de 24 años $( \pm 11)$. Se observó la aplicación de herbicidas selectivos, cuando ven alguna arvense de interés evitan rociarla. Si los campesinos cuentan con más de un terreno de siembra, pueden elegir en cuál no dañar las arvenses de interés y no ser selectivo en los otros terrenos. En dos de los casos los campesinos destinan una porción del terreno para promoción y cultivo de arvenses de interés como $B$. rapa y $B$. juncea.

\section{Conocimiento y consumo de arvenses}

A pesar del uso constante de herbicidas, el 93.9\% de los jefes de familia colectaron arvenses de la milpa el año pasado. El $63.6 \%$ cortan entre tres y seis especies de arvenses y el $36.4 \%$ entre una y tres. La mayoría de las arvenses las reconocieron por su nombre en tseltal y español. Todas las especies son percibidas en mayor o menor grado como espontáneas, pero $B$. juncea, $B$. rapa y $C$. longirostrata se siembran para consumo o venta (Tabla 1) y se consumen todo el año. La cantidad obtenida de la milpa de temporal puede no ser suficiente y recurren a otros medios de obtención, principalmente la compra, el solar y la milpa de riego (Tabla 2). Las especies $B$. juncea, B. rapa, C. longirostrata y P. philadelphica, son utilizadas de forma frecuente en la gastronomía local. Por ejemplo $C$. longirostrata se utiliza para elaboración de tamales y $P$. philadelphica para salsas que acompañan los alimentos.

Las familias que cuentan con siembra de riego y temporal poseen la ventaja, de que la milpa de riego se siembra en el mes de febrero y la de temporal en mayo o junio, pudiendo tener durante todo el año especies como $A$. hybridus, G. quadriradiata, $S$. oleraceus, O. corniculata y P. philadelphica.

En el acompañamiento a las labores del campo, se observó que los 10 jefes de familia consumen arvenses cuando descansan de las labores del campo. Por la facilidad de encontrarlas forman parte importante de su alimentación durante las la- 
Mascorro-de Loera et al.

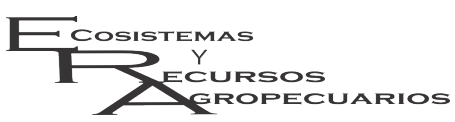

Tabla 2. Obtención y temporalidad de arvenses (porcentaje).

\begin{tabular}{lcccccc}
\hline \multirow{2}{*}{ Especie } & \multicolumn{2}{c}{ Temporalidad $^{a}$} & $\begin{array}{c}\text { Milpa de } \\
\text { temporal }\end{array}$ & Solar & $\begin{array}{c}\text { Milpa de } \\
\text { riego }\end{array}$ & Comprada \\
\cline { 2 - 6 } & $\mathrm{T}^{b}$ & $\mathrm{~A}^{c}$ & & & \\
\hline A. hybridus & 96.9 & 3.1 & 87.5 & 12.5 & 25.0 & 15.6 \\
G. quadriradiata & 97.0 & 3.0 & 87.9 & 3.0 & 24.2 & 3.0 \\
S. oleraceus & 81.8 & 27.3 & 93.9 & 12.1 & 27.3 & 15.2 \\
B. juncea & 40.6 & 59.4 & 39.4 & 0.0 & 9.1 & 90.9 \\
B. rapa & 39.4 & 60.6 & 45.5 & 9.1 & 6.1 & 84.8 \\
C. ambrosoides & 9.1 & 90.9 & 9.1 & 93.9 & 6.1 & 6.1 \\
I. purpurea & 50.0 & 25.0 & 25.0 & 0.0 & 25 & 0.0 \\
C. longirostrata & 36.4 & 63.6 & 30.3 & 3.0 & 3.0 & 81.8 \\
O. corniculata & 67.9 & 32.1 & 42.9 & 0.0 & 42.9 & 10.7 \\
P. oleracea & 76.2 & 23.8 & 71.4 & 19.0 & 19.0 & 0.0 \\
J. procumbens & 25.0 & 75.0 & 75.0 & 0.0 & 50.0 & 0.0 \\
S. nigrescens & 41.2 & 58.8 & 76.5 & 23.5 & 17.6 & 11.8 \\
P. philadelphica & 21.9 & 78.1 & 68.8 & 0.0 & 43.8 & 75.0 \\
\hline a Porcentaje obtenido con base en el número de familias que la consumieron el año \\
pasado o han consumido alguna vez, ${ }^{b}$ Temporal, se cosecha principalmente entre \\
junio y octubre, ${ }^{c}$ Anual, se puede conseguir en cualquier época del año.
\end{tabular}

bores de mantenimiento de la milpa. En caso de $C$. ambrosoides tiene poca presencia en la milpa pero se encuentra por lo general en el solar. Las mujeres se encargan de cuidarla, ya que es un condimento esencial en la preparación de alimentos.

La frecuencia de consumo de $A$. hybridus, G. quadriradiata, B. rapa, C. longirostrata y $P$. philadelphica ha disminuido, aunque las familias no han dejado de consumirlas. Lo contrario ocurre con O. corniculata, el porcentaje de familias que la consumen ha disminuido pero las que lo siguen haciendo no han modificado la frecuencia de consumo (Tabla 3). Aunque hay una tendencia a disminuir la frecuencia de consumo de arvenses, esto no se correlaciona con el tiempo de aplicación de herbicidas, ni el número de fumigaciones por ciclo de cultivo (Tablas 4 y 5$)$.

\section{DISCUSIÓN}

El manejo de la milpa en Amatenango del Valle ha sufrido cambios como lo reporta Castro-Ramírez y Silva (2002). Después de 1970 se volvió dependiente de los agroquímicos para mantener la milpa (Bernardino et al. 2014). Pero el uso del fuego, machete y azadón no se ha eliminado, posiblemente por la dificultad de eliminar algunas arvenses (Hernández 1985), que por medios químicos implican mayor costo y menor efectividad. Además, remover con azadón las arvenses alrededor de la planta de maíz permite su uso como abono (Mariaca et al. 2007). La aplicación de herbicidas es generalizado en Amatenango del Valle; ya que el $100 \%$ de los encuestados los usan, lo que indica un mayor uso que lo reportado para la misma comunidad (86.0\%) por Bernardino et al. (2014) o para los productores de maíz en el estado de Chiapas (93.3\%) (Eakin et al. 2014). También fue superior al $55.1 \%$ observado en la Zona Alta de San Felipe del Progreso, México (Nava et al. 1999), el $68 \%$ en Ixhuapán, Veracruz (Vázquez-García et al. 2004) y el $17.6 \%$ en Huautepec, Oaxaca (CarreraGarcía et al. 2012).

Las principales arvenses colectadas en la milpa de temporal fueron $A$. hybridus, $S$. oleraceus y G. quadriradiata. El consumo del género Amaranthus en México comenzó en la época prehispánica (Picó y Nuez 2000), siendo hoy en día $A$. hybridus una de las arvenses más consumidas (Albino-García et al. 2011, Ortíz-Timoteo et al. 2014) e importantes en la alimentación en zonas indígenas del país (Linares y Bye 2015) e incluso otros países (Madamombe-Myuna et al. 2009, Turreira-García et al. 2015). La especie S. oleraceus no es nativa del país (Vibrans 2009), pero se reporta su consumo en el estado de Puebla (Macín et al. 2013), lo que indica que las comunidades la han incorporado a su dieta. Al ser una especie exótica, existe mayor información de su consumo en Europa (Grundy et al. 2011, Keller et al. 2014, Molina et al. 
Mascorro-de Loera et al.

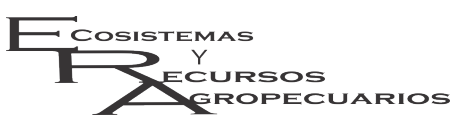

Herbicidas e impacto en la milpa

Ecosist. Recur. Agropec. 6(18):477-486,2019

Tabla 3. Frecuencia de consumo.

\begin{tabular}{|c|c|c|c|c|c|}
\hline \multirow{2}{*}{ Especie } & \multicolumn{2}{|c|}{ Frecuencia anual de consumo } & \multirow{2}{*}{$\mathrm{T}$} & \multirow{2}{*}{$\mathrm{gl}$} & \multirow[b]{2}{*}{$\mathrm{p}$} \\
\hline & Año pasado & Hace 10 años & & & \\
\hline A. hybridus & $8.75( \pm 9.68)$ & $10.19( \pm 10.65)$ & -2.757 & 30 & 0.010 \\
\hline G. quadriradiata & $8.24( \pm 7.82)$ & $11.21( \pm 11.83)$ & -2.462 & 32 & 0.019 \\
\hline S. oleraceus & $23.61( \pm 29.79)$ & $27.64( \pm 33.93)$ & -1.914 & 32 & 0.065 \\
\hline B. juncea & $22.76( \pm 20.02)$ & $25.79( \pm 26.34)$ & -1.529 & 30 & 0.137 \\
\hline B. rapa & $25.24( \pm 20.41)$ & $29.00( \pm 25.66)$ & -2.270 & 32 & 0.030 \\
\hline C. ambrosoides & $31.67( \pm 28.85)$ & $30.88( \pm 29.06)$ & 0.478 & 32 & 0.636 \\
\hline I. purpurea ${ }^{a}$ & $1.25( \pm 2.50)$ & $1.50( \pm 2.38)$ & - & - & - \\
\hline C. longirostrata & $6.58( \pm 8.28)$ & $7.76( \pm 9.42)$ & -2.730 & 32 & 0.010 \\
\hline O. corniculata & $2.39( \pm 4.80)$ & $4.04( \pm 6.16)$ & -1.000 & 13 & 0.336 \\
\hline P. oleracea & $5.67( \pm 6.80)$ & $6.76( \pm 8.97)$ & -1.268 & 18 & 0.221 \\
\hline J. procumbens ${ }^{b}$ & $3.25( \pm 4.57)$ & $3.50( \pm 4.35)$ & - & - & - \\
\hline S. nigrescens ${ }^{b}$ & $7.35( \pm 12.88)$ & $7.88( \pm 12.70)$ & - & - & - \\
\hline P. philadelphica & $48.82( \pm 31.61)$ & $55.52( \pm 32.94)$ & -2.140 & 31 & 0.040 \\
\hline
\end{tabular}

Tabla 4. Consumo de arvenses en relación con años desde la adopción de las herbicidas.

\begin{tabular}{cccc}
\hline \multirow{2}{*}{$\begin{array}{c}\text { Años de } \\
\text { aplicación de } \\
\text { herbicida en la milpa }\end{array}$} & \multicolumn{2}{c}{$\begin{array}{c}\text { ¿Come la misma cantidad de } \\
\text { "verduritas" que hace diez años? }\end{array}$} & Total \\
\cline { 2 - 3 } & lgual o más & Menos & \\
\hline $1-25$ & 12 & 7 & 19 \\
$>25$ & 8 & 3 & 11 \\
Total & 20 & 10 & $30^{\star}$ \\
\hline
\end{tabular}

$\mathrm{Chi}^{2}=0.287, \mathrm{gl}=1, \mathrm{p}=0.592,{ }^{*}$ Tres jefes de familia no recordaban cuantos años que llevan aplicando herbicidas.

Tabla 5. Consumo de arvenses en relación con el número de fumigaciones por ciclo de cultivo.

\begin{tabular}{cccc}
\hline \multirow{2}{*}{$\begin{array}{c}\text { Número } \\
\text { de }\end{array}$} & \multicolumn{2}{c}{$\begin{array}{c}\text { Come la misma cantidad de } \\
\text { "verduritas" que hace diez años? }\end{array}$} & Total \\
\cline { 2 - 3 } fumigaciones & lgual o más & Menos & \\
\hline 2 & 3 & 2 & 5 \\
3 & 17 & 9 & 26 \\
4 & 2 & 0 & 2 \\
Total & 22 & 11 & 33 \\
\hline
\end{tabular}

$\mathrm{Chi}^{2}=1.119, \mathrm{gl}=2, \mathrm{p}=0.571$.

2014). Mientras que Madamom-be-Myuna et al. (2009) reportaron su consumo en Zimbabwe. Para G. quadriradiata hay reportes de su consumo en otras partes del país (Vieyra-Odilon y Vibrans 2001, Sánchez-Blanco y Guevara-Féfer 2013).

La combinación del manejo manual con herbicidas practicado en Amatenango del Valle parece crear condiciones adecuadas para mantener la diversidad y presencia de arvenses. Al respecto se sabe que el uso del machete y azadón puede provocar dispersión inconsciente de semillas (Bye 1981). La aplicación selectiva de herbicida, la promoción de especies en zonas libres de herbicidas y la protección de individuos para asegurar la dispersión de semilla para el siguiente ciclo agrícola, lo que coincide con el manejo descrito por Caballero y Cortés (2001) que permite la adaptación de las especies en los agroecosistemas; consiste en la recolección, tolerancia, fomento o inducción y protección de las plantas de interés. Los estudios indican que hay menor diversidad de especies cuando la labranza se realiza con mecanismos invasivos como el tractor (Mavunganidze et al. 2014 Armengot et al. 2016).

El uso prolongado de herbicidas genera resistencia, fenómeno documentado para algunas especies del género Amaranthus (Heap 2014, Keller et al. 2014) y para S. oleraceus (Grundy et al. 2011). Por lo que es posible que este fenómeno explique en parte la presencia de estas especies en la milpa, además de ser dos de las más consumidas por las familias. Es posible que en Amatenango del Valle se esté presentando un cambio de alimentación como lo reportaron Álvarez et al. (2009) para el municipio de Comitán, Chiapas, donde el consumo de alimentos procesados está incrementando. Los encuestados mencionan que el consumo de $O$. corniculata ha sido sustituido por alimentos procesados. Esta arvense hervida con azúcar se utiliza como postre para los niños, pero el consumo de golosinas procesadas ha ocasionado que menos familias la consuman.

La diversidad de arvenses en Amatenango del 
Valle permite que las familias decidan cuáles consumir. Por lo que la tradición cultural (Bourges et al. 2015, McClung de Tapia et al. 2014), sabor (Bye y Linares 2000, Mera et al. 2005) o aroma (Bye y Linares 2000) son aspectos que influyen en el consumo de arvenses. Según los encuestados el consumir arvenses no está en función de escasez de otro tipo de alimentos como lo menciona Bye (1981) o como alimentos de emergencia (Vázquez-García et al. 2004). Debido a que las arvenses forman parte de la cultura gastronómica local, como se manifiesta en su persistencia en la gastronomía de Amatenango del Valle, a pesar de los cambios en el manejo de la milpa.

\section{CONCLUSIONES}

El uso de herbicidas se ha generalizado en la comunidad, todos los agricultores encuestados lo utilizan como método para el control de arvenses, de forma separada o en conjunto con el machete o azadón, y la quema; lo que posiblemente ayude a mantener la presencia y disponibilidad de arvenses comestibles. Las estrategias desarrolladas por los jefes de familia para proteger y promover su crecimiento como la aplicación selectiva de herbicidas, los refugios libres de herbicidas en la milpa, la colecta en los solares, en cultivos de riego y la compra, aseguran la continuidad de las tradiciones gastronómicas de la comunidad. La frecuencia de consumo de arvenses se encuentra en función de la decisión del jefe de familia, además las fuentes de obtención que no sea la milpa permiten tener disponibilidad durante gran parte del año. Las arvenses cuya frecuencia de consumo no ha variado muestra que culturalmente son importantes, para la gastronomía local.

\section{AGRADECIMIENTOS}

Al CONACyT por la beca económica otorgada para los estudios de posgrado.

\section{LITERATURA CITADA}

Albert LA (2005) Panorama de los plaguicidas en México. http://www.sertox.com.ar/retel/default.htm. Fecha de consulta: 22 de febrero de 2015.

Albino-García C, Cervantes H, López M, Ríos-Casanova L, Lira R (2011) Patrones de diversidad y aspectos etnobotánicos de las plantas arvenses del valle de Tehuacán-Cuicatlan: El caso de San Rafael, municipio de Coxcatlán, Puebla. Revista Mexicana de Biodiversidad 82: 1005-1019.

Álvarez, G, Eroza JE, Ramírez E (2009) A socio-cultural diagnosis of adolescent diets in Comitán, Chiapas. Social Medicine 4: 32-47.

Armengot L, Blanco-Moreno JM, Bàrberi P, Bocci G, Carlesi S, Aendekerk R, Berner A, et al. (2016) Tillage as a driver of change in weed communities: a functional perspective. Agriculture, Ecosystems y Environment 222: $276-285$.

Bernardino HU, Mariaca R, Nazar DA, Álvarez-Solís JD, Torres A, Herrera C (2014) Los plaguicidas en los altos de Chiapas: soluciones que matan. San Cristóbal de las Casas, Chiapas. El Colegio de La Frontera Sur. $168 p$.

Blanckaert I, Vancraeynest K, Swennen RL, Espinosa-García FJ, Piñero D, Lira-Saade R (2007) Non-crop resources and the role of indigenous knowledge in semi-arid production of Mexico. Agriculture, Ecosystems and Environment 119: 39-48.

Bourges H, Zubirán S, Vargas LA (2015) La cocina tradicional y la salud. Revista Digital Universitaria 16(5). Art 36. http://www.revista.unam.mx/vol.16/num5/art36/art36.pdf. Fecha de consultado: 15 de abril de 2017.

Buenrostro M (2009) Las bondades de la milpa. Ciencias 92-93: 30-32. 
Bye (1981) Quelites: ethnoecology of edible greens, past, present and future. Journal of Ethnobiology 1: 109123.

Bye R, Linares E (2000) Los quelites, plantas comestibles de México. Una reflexión sobre intercambio cultural. Biodiversitas 31: 11-14.

Caballero J, Casas A, Cortés L, Mapes C (1998) Patrones en el conocimiento, uso y manejo de plantas en pueblos indígenas de México. Revista de Estudios Atacameños 16: 181-196.

Caballero J, Cortés L (2001) Percepción, uso y manejo tradicional de los recursos vegetales en México. En: Rendón B, Rebollar S, Caballero J, Martínez MA (ed). Plantas, cultura y sociedad. Estudios sobre la relación entre seres humanos y plantas en los albores del siglo XXI. UAM/SEMARNAP. México. pp: 79100.

Caballero A, Ayora T, Dumani M, Escobar D (2011) Los recursos vegetales en la alimentación de mujeres tsotsiles de la Selva El Ocote, Chiapas, México. Lacandonia 5: 141-147.

Carrera-García S, Navarro-Garza H, Pérez-Olvera MA, Mata-García B (2012) Calendario agrícola mazateco, milpa y estrategia alimentaria campesina en territorio de Huautepec, Oaxaca. Agricultura, Sociedad y Desarrollo 9: 455-475.

Castro-Ramírez A, Silva M (2002) Hacia la producción sustentable de maíz de temporal en los Altos de Chiapas. En: López-Olguín JF, Tornero M (ed). Métodos para la generación de tecnología agrícola de punta, Benemérita Universidad Autónoma de Puebla. Puebla. BUAP. pp: 159-170

Chikoye D, Schulz S, Ekeleme F (2004) Evaluation of integrated weed management practices for maize in the northern Guinea savanna of Nigeria. Crop Protection 23: 895-900.

Eakin H, Perales H, Appendini K, Sweeney S (2014) Selling Maize in Mexico: The Persistence of Peasant Farming in an Era of Global Markets. Development and Change 45: 133-55.

González-Amaro RM, Martínez-Bernal A, Basurto-Peña F, Vibrans H (2009) Crop and non-crop productivity in a traditional maize agroecosystem of the highly of Mexico. Journal of ethnobiology and ethnomedicine 5 (38) Doi:10.1186/1746-4269-5-38.

Grundy AC, Mead A, Bond W, Clark G, Burston S (2011) The impact of herbicide management on long-term changes in the diversity and species composition of weed populations. Weed Research 51: 187-200.

Heap I (2014) Global perspective of herbicide-resistant weeds. Pest Management Science 70: 1306-1315.

Hernández E (1985) La agricultura en la Península de Yucatán. En: Xolocotzia. Obras de Efraím Hernández Xolocotzi. Tomo I. Revista de Geografía Agrícola. México: Universidad Autónoma Chapingo. pp: 371-409

INEGI (2005) Prontuario de información geográfica municipal de los Estados Unidos Mexicanos. Amatenango del Valle, Chiapas. http://www3.inegi.org.mx/contenidos/app/mexicocifras/datos_geograficos/07/07007.pdf. Fecha de consulta: 12 de diciembre de 2017.

Keller M, Böhringer N, Möhring J, Rueda-Ayala V, Gutjahr C, Gerhards R (2014) Long-term changes in weed occurrence, yield and use of herbicides in maize in south-western Germany, with implications for the determination of economic thresholds. Weed Research 54: 457-66.

Ladio AH, Lozada M (2004) Patterns of use and knowledge of wild edible plants in distinct ecological environments: a case study of a Mapuche community from northwestern Patagonia. Biodiversity and Conservation 13: 1153-1173. 
Mascorro-de Loera et al.

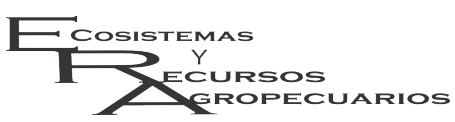

Herbicidas e impacto en la milpa

Ecosist. Recur. Agropec. 6(18):477-486,2019

Lara E, Caso L, Aliphat M (2012) El sistema milpa roza, tumba y quema de los Mayas Itzá de San Yrés y San José, Petén Guatemala. Ra Ximhai 8: 71-92.

Linares ME, Bye BR (2011) ¡La milpa no es solo maíz!. En: Álvarez-Buylla E, Carreón A, San Vicente A (ed). Haciendo milpa. Universidad Nacional Autónoma de México. México. pp: 9-12.

Linares ME, Bye BR (2015) Las especies subutilizadas de la milpa. Revista Digital Universitaria 16: 1-22.

Macín G, Gómez JA, Ballesteros M, Canizales S, Acuña V (2013) Cambio cultural, estilo de vida, adiposidad y niveles de glucosa en una comunidad totonaca de la Sierra Norte de Puebla. Revista Cuicuilco 58: 173-96.

Madamombe-Myuna I, Vibrans H, López-Mata L (2009) Diversity of coevolved weeds in smallholder maize Welds of Mexico and Zimbabwe. Biodiversity Conservation 18: 1589-1610.

Mariaca R, Pérez J, León NS, López A (2007) La milpa tsotsil de los Altos de Chiapas y sus recursos genéticos. México: El Colegio de La Frontera Sur/Universidad Intercultural de Chiapas. Chiapas, México. 272p.

Mavunganidze Z, Madakadze IC, Nyamangara J, Mafongoya P (2014) The impact of tillage system and herbicides on weed density, diversity and yield of cotton (Gossipium hirsutum L.) and maize (Zea mays L.) under the smallholder sector. Crop Protection 58: 25-32.

McClung de Tapia E, Martínez D, Ibarra E, Adriano CC (2014) Los orígenes prehispánicos de una tradición alimentaria en la cuenca de México. Anales de Antropología 48: 97-121.

Mera LM, Flores R, Basurto-Peña F, Bye R, Castro D, Evangelista V, Mapes C, et al. (2005) De quelites me como un taco. Ciencias 77: 36-38.

Molina M, Tardío J, Aceituno-Mata L, Morales R, Reyes-García V, Pardo-de-Santayana M (2014) Weeds and food diversity: Natural yield assessment and future alternatives for traditionally consumed wild vegetables. Journal of Ethnobiology 34: 44-67.

Nava EG, Arriaga C, Chávez MC (1999) La vegetación arvense en sistemas de producción campesinos de dos zonas del municipio de San Felipe del Progreso, México. Revista de Geografía Agrícola 29: 29-42.

Ortíz-Timoteo J, Sánchez-Sánchez OM, Ramos-Prado JM (2014) Actividades productivas y manejo de milpa en tres comunidades campesinas del municipio de Jesús Carranza, Veracruz, México. Polibotánica 38: 173-191.

Picó B, Nuez F (2000) Minor crops of Mesoamerica in early sources (I). Leafy vegetables. Genetic Resources and Crop Evolution 47: 527-540.

Ramírez-Salinas C, Castro-Ramírez AE (2011) 'Los montes', conocimiento tradicional campesino sobre las arvenses de la milpa en Teopisca y Amatenango del Valle, Chiapas. En: Ávila EL (ed). Desarrollo sustentable, interculturalidad y vinculación comunitaria Chiapas. Universidad Intercultural de Chiapas. Chiapas, México. pp: 95-117.

SIAP (2017) Amatenango del Valle, Chiapas. Servicio de Información Agroalimentaria y Pesquera. http://infosiap. siap.gob.mx/aagricola_siap_gb/icultivo/index.jsp. Fecha de consulta: 9 de junio de 2017.

Sánchez-Blanco J, Guevara-Féfer F (2013) Plantas arvenses asociadas a cultivos de maíz de temporal en suelos salinos de la rivera del lago de Cuitzeo, Michoacán, México. Acta Botanica Mexicana 105: 107-29.

Silva M, Castro-Ramírez AE, Cortés JL, Ishiki M (2003) Entomofauna asociada a maíz de temporal con diferentes manejos de malezas en Chiapas, México. Manejo Integrado de Plagas y Agroecología 70: 65-73.

Sinha R, Lakra V (2007) Edible weeds of tribals of Jharkhy, Orissa and West Bengal. Indian Journal of Traditional Knowledge 6: 217-222. 
Solís-Becerra CG, Estrada-Lugo EIJ (2014) Prácticas culinarias y (re)conocimiento de la diversidad local de las verduras silvestres en el Colectivo Mujeres y Maíz de Teopisca, Chiapas, México. Revista LaminaR. Estudios Sociales y Humanísticos 12: 148-162.

Sonnenfeld D (1992) Mexico's 'green revolution', 1940-1980: Towards an environmental history. Environmental History Review 16: 28-52.

Turreira-García N, Theilade I, Meilby H, Sørensen M (2015) Wild edible plant knowledge, distribution and transmission: a case study of the Achí Mayans of Guatemala. Journal of Ethnobiology and Ethnomedicine 11:52. Doi:10.1186/s13002-015-0024-4.

Vázquez-García V, Godínez-Guevara L, Montes-Estrada M, Montes-Estrada M, Ortíz-Gómez AS (2004) Los quelites de Ixhuapan, Veracruz: disponibilidad, abastecimiento y consumo. Agrociencia 38: 445-455.

Vibrans H (2009) Ficha - Sonchus oleraceus. Malezas de México. http://www.conabio.gob.mx/malezasdemexico/ asteraceae/sonchus-oleraceus/fichas/ficha.htm. Fecha de consulta: 2 de junio de 2017.

Vieyra-Odilon L, Vibrans H (2001) Weeds as Crops: the value of maize field weeds in the Valley of Toluca, Mexico. Economic Botany 55: 426-443.

Vigouroux Y, Barnaud A, Scarcelli N, Thuillet AC (2011) Biodiversity, evolution and adaptation of cultivated crops. Comptes Rendus - Biologies 334: 450-457.

Viveros AD (2005) Química y ecotoxicología de los herbicidas. En: Botello JAV, Rendón-von O, Gold-Bouchot C, Agraz-Hernández C (ed). Golfo de México contaminación e impacto ambiental: Diagnóstico y tendencias. $2^{a}$ edición. UACAM, UNAM, Instituto Nacional de Ecología. México. pp: 199-206.

Zizumbo-Villarreal D, Colunga-García P (2010) Origin of agriculture and plant domestication in West Mesoamerica. Genetic Resources y Crop Evolution 57: 813-825. 